\title{
La correspondance de Georges Lefebvre avec l'historien Gordon H. McNeil (1940-1954)
}

James Friguglietti

\section{(2) OpenEdition \\ 1 Journals}

Édition électronique

URL : https://journals.openedition.org/ahrf/11537

DOI : 10.4000/ahrf.11537

ISSN : 1952-403X

Éditeur :

Armand Colin, Société des études robespierristes

\section{Édition imprimée}

Date de publication : 1 octobre 2009

Pagination : 133-142

ISBN : 978-2-200-92560-4

ISSN : 0003-4436

\section{Référence électronique}

James Friguglietti, « La correspondance de Georges Lefebvre avec l'historien Gordon H. McNeil

(1940-1954)", Annales historiques de la Révolution française [En ligne], 358 | octobre-décembre 2009

mis en ligne le 01 octobre 2012, consulté le 24 avril 2022. URL : http://journals.openedition.org/ahrf/ 11537 ; DOl : https://doi.org/10.4000/ahrf.11537 
GEORGES LEFEBVRE :

\section{POUR LE CINQUANTIÈME ANNIVERSAIRE DE SA MORT}

\section{LA CORRESPONDANCE DE GEORGES LEFEBVRE AVEC ROBERT R. PALMER (1948-1959)}

James FRIGUGLIETTI

Disparu le 28 août 1959 à l'âge de 85 ans, Georges Lefebvre avait produit au cours de sa longue vie une quantité impressionnante de publications sur l'époque révolutionnaire et napoléonienne ${ }^{1}$. Pourtant, si son œuvre historique est bien connue, sa vie privée demeure obscure. Préférant rester discret quant à ses affaires personnelles, il ne se révélait que rarement, comme dans son bref essai « Pro domo », paru dans les Annales historiques de la Révolution française en $1947^{2}$.

À deux reprises, cependant, des extraits de sa correspondance ont été publiés dans cette même revue, le premier avec l'historien suisse Alfred Rufer en 1979³ , le deuxième avec l'érudit britannique J. M. Thompson en $2000^{4}$. Un troisième, présenté ici, fournit des détails sur le progrès de son travail, ses voyages, ses opinions politiques ainsi que l'état de sa santé. Adressées à son collègue et ami américain R. R. Palmer ${ }^{5}$,

(1) Sur son œuvre, voir James FriguglietTI, Bibliographie de Georges Lefebvre, Paris, Société des études robespierristes, 1972.

(2) $A H R F$, t. 19,1947 , p. $188-190$

(3) Ibid., t. 55, 1979, p. 430-442.

(4) Ibid., t. 72, 2000, p. 111-119.

(5) R. R. Palmer (1909-2002) est né à Chicago et a fait ses études supérieures à Cornell où, sous la direction de Carl Becker, il a rédigé sa thèse de doctorat sur l'opinion française à l'égard de la Révolution américaine. Il enseigna l'histoire à Princeton de 1936 à 1963, puis de 1966 à 1969. Parmi ses nombreux livres figurent Twelve Who Ruled. The Committee of Public Safety during the Terror, Princeton, Princeton University Press, 1941 (trad. fr. : Le Gouvernement de la Terreur. L'année 
traducteur de son livre Quatre-vingt-neuf, paru en anglais à la Princeton University Press en 1947 sous le titre The Coming of the French Revolution $^{6}$, ces vingt-trois lettres étalées sur la période 1948-1959 ne représentent qu'une partie de leur correspondance. Commencée avant l'invasion allemande, interrompue pendant l'occupation et reprise avec la Libération, la première partie de la correspondance à été malheureusement perdue. Aux textes qui subsistent, difficilement déchiffrables à cause de sa fine écriture, nous avons ajouté des notes qui servent à éclairer des références obscures.

Cet hommage à Georges Lefebvre rappellera à une nouvelle génération son importance dans l'historiographie de la Révolution et de Napoléon. Il pourra l'inspirer aussi en montrant comment cet historien engagé poursuivit son travail malgré la vieillesse et dans les conditions difficiles de l'après-guerre.

Je tiens à remercier M. Julien Louvrier qui a lu le manuscrit avec le plus grand soin et a corrigé de nombreuses erreurs glissées dans la transcription de la correspondance. En plusieurs endroits, il a réussi à déchiffrer l'écriture chancelante de Georges Lefebvre. Je lui suis reconnaissant de son aide précieuse.

James FrigugLIETTI

Montana State University-Billings

Billings, Montana

USA

jfriguglietti@msubillings.edu

(Suite de la note 5 page 121)

du Comité de salut public, Paris, A. Colin, 1989), et The Age of the Democratic Revolution. A Political History of Europe and America, 1760-1800, Princeton, Princeton University Press, 1959-1964, 2 vol. (trad. fr. : 1789. Les révolutions de la liberté et de l'égalité, Paris, Calmann-Lévy, 1968). Sur la vie et l'œuvre de R. R. Palmer, voir $A H R F$, t. 74, 2002, p. 159-163.

(6) Une nouvelle édition revue et corrigée par Timothy Tackett a été publiée par Princeton University Press en 2005. 
Boulogne s/S, 29-3-48

Cher ami,

J'ai reçu votre lettre du 23. J'y réponds sans tarder, je l'avoue, parce que j'étais sur le point de vous écrire à nouveau pour vous consulter sur une question qui intéresse notre préparation du Recueil de documents relatifs aux États-généraux de $1789^{1}$. Le premier volume qui s'achève, contient, entre autres, les procès-verbaux des conférences relatives à la vérification des pouvoirs. L'édition critique que nous avons préparée a mis en lumière le grand rôle qu'y a joué Dupont de Nemours. Comme vous le savez la famille qui descend de lui compte aujourd'hui parmi les plus connues et les plus puissantes de votre pays. Or, elle conserve des papiers de l'ancêtre. Quand [Gustave] Schelle publia en 1886 son livre sur Turgot ${ }^{2}$ il signala que les descendants américains de Dupont possédaient plus de 300 lettres de Turgot à ce dernier. Il est possible que des papiers relatifs à l'activité de Dupont aux États-généraux fassent partie de leur collection.

Malheureusement, Schelle ajoute que la communication des lettres de Turgot lui a été refusée. Mais depuis Mme. B[essie Gardner] Dupont de Nemours a écrit une vie de Dupont durant son incarcération au temps de la Terreur. [Gilbert] Chinard ${ }^{3}$ a obtenu de Mme. Dupont et Mr. P[ierre] Dupont de Nemours l'autorisation de les reproduire et en a donné une édition française. Nous pensons donc qu'il vaudrait la peine de faire une tentative pour obtenir des renseignements sur l'existence éventuelle des papiers de Dupont relatifs aux États-généraux et peut-être en obtenir communication. À ce que je sais, Mr. Dupont de Nemours est installé à Wilmington [Delaware]. Puis-je demander des instructions sur la manière de procéder à la démarche ? Si, par extraordinaire, on consentait à nous ouvrir ces archives, consentiriez-vous à aller les examiner pour nous ? En ce cas, les dollars qu'éventuellement vous tiendriez à réserver à ma disposition pour les royalties du Coming of the French Revolution seraient tout indiqués pour commencer à vous couvrir de vos frais.

J'ai remercié la société Mark Twain : nous verrons ce qui en résultera $^{4}$.

Je suis content d'apprendre que la situation économique des Étatsunis ne vous paraît pas inquiétante. Les préoccupations ne sont pourtant pas entièrement dissipées. Elles résultent en partie de vues de principe sur le caractère inhérent au capitalisme d'engendrer des crises de plus en plus fréquentes et de plus en plus redoutables à mesure que sa capacité produc- 
trice augmente. C'est un des points de l'analyse de Marx le mieux d'accord avec la nature des choses et le mieux confirmé par l'expérience historique. Pour m'en tenir à un point actuel, on allègue que le pouvoir d'achat des masses américaines est resté jusqu'à présent supérieur à la normale parce que, durant la guerre, elles ont épargné beaucoup, les salaires étant abondants, le chômage supprimé et nombre de marchandises consommables n'étant pas mises à leur disposition puisque l'industrie était principalement occupée par les fabrications de guerre. Mais cette épargne doit s'épuiser et les prix montent; on doit donc s'attendre à voir reparaître l'impossibilité pour la masse salariée d'acheter ce qu'elle a produit puisque le profit capitaliste ne permet de lui servir comme salaire qu'une partie de la valeur produite. En ce cas, l'exportation est pour le capitalisme une nécessité inéluctable, et comment exporter si, hors des États-Unis, personne n'est en état de payer? Je m'excuse de n'en pas dire d'avantage parce qu'il y faudrait encore bien des pages.

Pour ce qui concerne la France, je vois dans votre lettre que je ne suis pas seul à douter que la politique du gouvernement actuel puisse suffire à restaurer notre économie, nos finances et notre monnaie.

Quant à De Gaulle, ses ambitions napoléoniennes paraissent probables. Elles sont pure chimère. Mais s'il arrive au pouvoir, il n'a pas caché qu'il lierait partie avec ceux qui, chez vous, envisagent comme inévitable et nécessaire une troisième guerre mondiale. Évidemment le danger ne vient pas de lui qui n'a pas de moyens : il réside dans la politique américaine, car, dans mon opinion, la Russie est actuellement incapable de souhaiter la guerre; elle est encore trop dévastée et trop imparfaitement industrialisée pour cela. Ce qui est au juste la politique américaine, je ne le sais pas. Je ne doute pas que les intellectuels et le peuple répugnent à la guerre ; mais il ne manque pas d'indices que certains éléments en envisagent le risque sans trembler et leur application à répandre la peur peut réussir à entraîner les autres surtout si une crise économique se déclarait. Voilà quelques-unes des raisons de notre anxiété. Je souhaite de tout cœur qu'elles ne soient pas fondées.

\section{Bien amicalement vôtre}

\section{Lefebvre}

P. S. Je vous remercie une fois de plus pour ce qui concerne les royalties. Il me semble que le mieux pour le moment est que vous gardiez en réserve tout ce qu'il vous conviendra de m'attribuer. 
(1) Recueil de documents relatifs aux séances des États-généraux, mai-juin 1789. Préparé par l'Institut d'histoire de la Révolution française de la Faculté des lettres de Paris, sous la direction de Georges Lefebvre et Anne Terroine. T. I : Les préliminaires. La séance du 5 mai, Paris, Centre national de la recherche scientifique, 1953.

(2) Gustave Schelle (1845-1927), Du Pont de Nemours et l'École physiocratique, Paris, Guillaumin, 1888 (G. Lefebvre s'est trompé sur la date de publication). Dans ce livre, Schelle se réfère à maintes reprises à la vie et à l'œuvre de Turgot.

(3) Gilbert Chinard (1881-1972), né à Châtellerault (Vienne), a fait ses études universitaires à Bordeaux et à la Sorbonne. Dès 1908, il s'installa aux États-Unis où il enseigna le français dans diverses universités, avant de devenir professeur de littérature française à Johns Hopkins en 1919. À partir de 1937, il enseigna à Princeton où il devait rester jusqu'à sa retraite en 1950. C'est là qu'il devint un ami de R. R. Palmer. Pendant sa longue carrière il publia une cinquantaine de livres, surtout consacrés aux relations franco-américaines, dont des études sur Thomas Jefferson et Benjamin Franklin. Parmi ses publications sur la Révolution française figure son édition des Lettres de Dupont de Nemours écrites de la prison de la Force, 5 thermidor-8 fructidor an II, Paris, Librairie historique A. Margoff, 1929, $102 \mathrm{p}$.

(4) La Mark Twain Society, fondée aux États-Unis en 1927, invitait des gens de lettres de tous pays à y adhérer. G. Lefebvre semble avoir refusé une invitation sans conséquence.

Boulogne s/S, 29-4-48

86 , Bd Jaurès

Cher ami,

Je ne sais comment vous remercier convenablement du soin que vous prenez de me ravitailler ${ }^{1}$. Veuillez bien vous persuader de ma gratitude. Je vais me mettre en rapport avec Chinard et je veux espérer que nos efforts convergents parviendront à un résultat satisfaisant.

Les nouvelles que vous me donnez du débit de votre traduction comblent mes expériences comme celles de Mr. Datus Smith². Son nom, que vous citez, m'a rappelé qu'il vous avait offert de m'envoyer quelquesuns des volumes publiés par la Princeton University Press. Je serais contrarié s'il pouvait penser que je n'ai pas pris en due considération sa proposition. Mais, comme je crois vous l'avoir écrit, je n'ai pas retrouvé la liste de vos publications. Je me souviens seulement de l'Histoire des États-Unis de Wilson ${ }^{3}$ qui d'ailleurs doit bien probablement se trouver à présent épuisée. Si vous vouliez bien m'envoyer le prospectus contenant la liste de vos publications, je me rappellerais de nouveau à votre bienveillance à cet égard.

Je comprends fort bien que le résultat des élections italiennes vous ait procuré un sentiment de satisfaction en ce qu'il ajourne les complications que nous redoutons. Vous avez raison de craindre que l'intervention 
évidente des États-Unis dans ces élections ne laisse pas de troubler l'opinion - du moins parmi les intellectuels. On en vient à penser que l'Europe occidentale qu'on travaille à construire deviendra inévitablement une sorte de nouvelle Sainte Alliance et on n'est pas loin de regarder $\mathrm{M}^{\mathrm{r}}$. Truman comme un nouveau Metternich. En tout cas, ce qu'il y a d'incontestable, c'est que la démocratie, telle que les États-Unis tendent maintenant à la définir - et avec les États-Unis, les anti-communistes de l'Europe sont clairement d'accord - est telle que la concevaient beaucoup d'hommes de 1789 et Sieyès lui-même tout au moins en l'an III. Le suffrage universel et la souveraineté du peuple sont la base de cette démocratie mais à la condition qu'il n'en résulte aucun dommage pour ce que la classe dominante considère comme des droits antérieurs et supérieurs à la société spécialement le droit de propriété tel qu'il est actuellement compris et sanctionné par la loi. Si le suffrage universel paraît susceptible de prétendre à la modifier, on est disposé à le lui interdire, même par la force.

Ce n'est pas ainsi que les républicains démocrates concevaient la démocratie dans ma jeunesse. Les socialistes français et Jaurès notoirement, non plus. Ils ne bornaient pas l'évolution et se contentaient de recommander de renoncer à la violence et de procéder par la propagande rationnelle et pacifique. En ce cas, si le suffrage universel venait à se prononcer pour l'économie dirigée ou pour le communisme, il ne ferait qu'user du droit démocratique.

Si Marx avait à juger l'interprétation contraire qui semble prévaloir, il n'y a pas de doute qu'il y verrait l'effet logique de la lutte de classes et la preuve qu'elle est le moteur essentiel de l'histoire.

Je serais obligé de lui donner raison. Et la conclusion n'est pas consolante : c'est que l'humanité ne semble pas plus qu'autrefois disposée à renoncer au conflit menaçant pour accepter de s'en rapporter à l'évolution pacifique. Car si l'on prétend la suspendre au besoin par la force comme chez nous prétendit le faire [François] Guizot, l'espoir me paraît bien mince.

Bien amicalement vôtre

Lefebvre

(1) De temps en temps, Palmer expédiait à G. Lefebvre des colis de nourriture.

(2) Datus Smith (1907-1999), directeur de la Princeton University Press de 1941 à 1950.

(3) Woodrow Wilson, A History of the American People, 5 vol., New York, Harper \& Brothers, 1902. 
Mon cher collègue,

J'ai communiqué votre lettre à Chinard. Il n'a pas été surpris de l'accueil réticent qu'on nous fait à Wilmington. De retour aux États-Unis en octobre, il manifeste l'intention d'entrer en pourparlers avec $M^{r}$. Dupont de Nemours qui a écrit une biographie des membres de la famille ${ }^{1}$ et vous faire part du résultat. J'estime d'ailleurs que ces archives ne doivent rien posséder qui nous soit utile. $\mathrm{M}^{\mathrm{lle}}$ [Anne] Terroine ${ }^{2}$, qui travaille avec moi au Recueil [de documents relatifs aux séances] des États-généraux, compte pouvoir faire photographier un autographe de Dupont que nous enverrons soit à Dupont de Nemours, soit à vous-même, selon ce que vous jugerez préférable. Cela les rendra peut-être plus complaisants.

J'ai été fort content de l'accueil qu'a reçu votre traduction. On a beaucoup moins parlé de mon livre en France qu'aux États-Unis. On n'est jamais prophète en son pays, a-t-on dit ! J'ai reçu une demande de Stockholm : je suppose que c'était pour obtenir la faculté de vendre votre traduction et non pour exécuter une version en suédois. J'ai invité l'éditeur, dans la première hypothèse, à s'adresser à la Princeton [University] Press et j'espère qu'il l'a fait. On parle aussi d'une traduction en italien ${ }^{3}$ et en allemand (en Suisse).

Les nouvelles continuent d'être peu réconfortantes, mais je ne m'étends pas attendu que je vais partir pour le Midi où ma fille ${ }^{4}$ se repose après une crise cardiaque qui l'a tenue deux mois à l'hôpital. Je rentrerai à la fin du mois pour reprendre le travail.

J'ai bien reçu vos catalogues de la Princeton [University] Press. S'il est toujours dans l'intention de votre Directeur de m'envoyer les volumes dont je joins l'indication, je lui en serais bien reconnaissant. À sa discrétion, bien entendu, et sans être indiscret.

\section{Bien affectueusement,}

\section{Lefebvre}

(1) Pierre S. Du Pont, Genealogy of the Du Pont Family, 1739-1942, Wilmington, Hambleton, 1943 .

(2) Anne Terroine (1909-1976), archiviste-paléologue. Sur sa carrière voir l'article nécrologique publié dans les $A H R F$, t. 50, 1978, p. 156-157.

(3) L'Ottantanove, traduction d'A. Galante Garrone, Turin, G. Einaudi, 1949.

(4) Il s'agit de sa fille aînée, Marie-Louise-Victorine, née à Cherbourg en 1899, décédée à Paris en 1953. Voir la lettre en date du 22 juin 1953. 
Boulogne s/S, 5 septembre 1948

\section{Cher ami,}

J'ai attendu pour vous écrire. L'arrivée des livres que l'Université de Princeton a eu la libéralité de m'envoyer. Ils me sont parvenus en effet en trois paquets qui étaient intacts. J'ai remercié $\mathrm{M}^{\mathrm{r}} \mathrm{Busch}$ [?] qui m'avait annoncé l'expédition en observant que le volume de Strayer, Interpretation of History ${ }^{1}$, qui me piquait spécialement ma curiosité, et qu'il citait dans sa lettre, ne s'était pas trouvé avec les autres.

Je crains bien que nous n'obtiendrions rien des Dupont de Nemours. Néanmoins, j'ai remis à Chinard deux photographies des autographes de leur ancêtre ; il compte entrer en rapport avec la dame qui a écrit la biographie de divers membres de la famille.

Les catalogues de [Gérard] Walter ${ }^{2}$ si mal faits et confus qu'ils soient, peuvent évidemment rendre des services. Mais il est déplorable que le Directeur de la Bibliothèque nationale ait approuvé cette entreprise : à lui seul, un homme ne pouvait pas l'exécuter convenablement, surtout si vite. Elle a coûté beaucoup d'argent et, comme vous l'avez vu par l'article de $\mathrm{M}^{\mathrm{lle}}$ [Anne] Terroine ${ }^{3}$, risque d'induire les travailleurs à des erreurs et a des omissions. On ne sait d'ailleurs qui est ce Walter. Walter doit être un nom de guerre; il n'est pas français et n'a pas de documents d'identité car la Bibliothèque nationale n'a pas de dossier à son nom et n'a pu le titulariser.

L'envoi de [la] Princeton [University] Press comprenait cinq exemplaires de votre ouvrage. Je me propose de les remettre à des libraires. Clavreuil, qui est spécialisé dans les ouvrages sur la Révolution, dans la rue $\mathrm{S}^{\mathrm{t}}$ André des Arts, a accepté, mais il demande que vous lui fixiez un prix en francs; il prendra une commission de $33 \%$. Nous pourrions nous arranger, s'ils se vendent, pour remettre le produit à un de vos compatriotes qui l'utiliserait pendant son séjour en France et vous le restituerait en dollars à son retour. Il me semble que c'est possible.

Il n'est plus rien venu de Stockholm. Le correspondant songeait vraisemblablement à vendre l'édition anglaise. Mais, en ce cas, je m'étonne qu'il ne soit pas entré en rapport avec Princeton [University] Press.

En juillet, nous nous sommes demandés ici si la crise de Berlin ne finirait pas par provoquer la guerre. Mais les choses paraissent s'arranger pour le moment. Pour le moment seulement, hélas ! J'ai parlé de notre situation avec [Gilbert] Chinard et plusieurs de vos collègues qui passent 
quelques temps chez nous. Votre élection présidentielle jouera certainement un grand rôle dans la suite des événements. Elle nous laisse perplexes. Mais notre situation politique n'est pas plus claire. Vous savez déjà que la combinaison Marie-Reynaud ${ }^{4}$ n'a pas réussi. Elle avait pour but de revenir à la liberté économique complète et de faire supporter la hausse des prix qui en résultera nécessairement aux salariés en refusant une hausse correspondante de leurs rémunérations. Les socialistes ont regimbé et il était logique que, pour pratiquer pareille politique, on les expulse à leur tour de la majorité. Ils devaient s'y attendre et n'ont pas à se plaindre. Mais les catholiques M.R.P. ${ }^{5}$ (je ne parle pas du clergé) n'ont pas voulu s'abandonner au capitalisme en se séparant des socialistes et tout a été remis en question. Je pense que les socialistes vont comme toujours capituler moyennant quelques concessions : une hausse légère des salaires qui fera traîner de nouveau les choses pendant quelques semaines. Après quoi, tout recommencera car l'inflation continue. Pour y mettre fin il faudrait diminuer les dépenses militaires et il n'en est pas question.

Ma fille va un peu mieux mais n'a pas pu reprendre encore son service. Chez moi, rien de nouveau. J'espère que, de même, votre existence est restée paisible.

Bien affecteusement vôtre

\section{Lefebvre} 1943, 186 p.

(1) Joseph Strayer, The Interpretation of History, Princeton, Princeton University Press,

(2) Gérard Walter (1896-1974), bibliographe et historien. Éditeur, avec André Martin, du Catalogue de l'histoire de la Révolution française, 6 tomes en 7 volumes, Paris, Éditions des Bibliothèques nationales, 1936-1969.

(3) A. Terroine, « L'CEuvre bibliographique de M. G. Walter », AHRF, t. 19, 1947, p. 1-26.

(4) Ministère formé le 26 juillet 1948 et dissous le 5 septembre de la même année. Président du conseil : André Marie, ministre des Finances et affaires économiques : Paul Reynaud.

(5) Mouvement républicain populaire. 
Boulogne s/S, 20-10-48

Cher ami,

La Princeton [University] Press m'a en effet annoncé que l'ouvrage de [Joseph] Strayer était épuisé. $\mathrm{M}^{\mathrm{r}}$ Busch [?] a ajouté, comme vous me l'annonciez, qu'elle en rechercherait un exemplaire.

Le succès de votre traduction dépasse mes prévisions et je vous en félicite. Je suis touché plus que jamais du soin que vous prenez de m'associer au profit pécuniaire. Je suis tout à fait d'avis que $\$ 110$ dépassent de beaucoup ce qui pourrait être employé en colis. Quant à l'envoi d'argent, il m'embarrasse. On vient de dévaluer le franc et ce ne sera malheureusement pas la dernière fois. Ne seriez-vous pas d'opinion qu'il vaudrait mieux que vous gardiez ces dollars pour le moment ? Jusqu'à nouvel ordre, je ne suis pas en besoin urgent de cette ressource et les francs que je recevrais risquent de s'évaporer sans avantage par suite de la hausse précipitée. Il me semble qu'il est bien préférable que les dollars restent en vos mains.

L'aventure de Gérard Walter est bien digne d'étonner. Mais c'est ainsi. Il n'est pas un inconnu en ce sens qu'il nous inonde de volumes aux sujets les plus divers. Mais sur sa provenance, ses titres, et même son nom exact, c'est le mystère.

La situation ici reste instable et inquiétante. Celle du monde n'est guère meilleure. On attend votre élection présidentielle pour y voir plus clair. On avait beaucoup attendu du plan Marshall, mais la désillusion fait de grand progrès. Faute de pouvoir vous promettre un avenir souriant, je vous souhaite bon travail.

Bien amicalement vôtre

Lefebvre

Boulogne s/S, 23-1-49

Cher ami,

Je suis confus de vous répondre si tardivement pour vous remercier de votre envoi de Noël et de vos vœux. J'ai vu avec plaisir que vos deux 
fils paraissent prospères. Mais je sais par une expérience ancienne (mes deux filles viennent d'atteindre 49 et 48 ans) combien ils doivent prendre de temps à leur maman et à vous-même. J'ai attendu l'arrivée de vos deux colis pour vous écrire. Mais la grippe qui sévit ici a obscurci bientôt ma pensée.

J'ai été atteint de congestion pulmonaire ; on m'a mis sous sulfamides avec défense de sortir.

La fièvre et les sulfamides m'ont plongé dans l'hébétement assez longtemps. Je commence à reprendre mes sens, mais la force me manque encore.

L'un de vos colis vient de nous arriver. Il a été gravement endommagé pendant la traversée paraît-il ; la poste du moins l'assure. Elle a dû refaire l'emballage et j'y ai trouvé la note ci-jointe que je vous communique à titre de curiosité et en vous renouvelant mes remerciements.

La Bibliothèque de la Sorbonne m'a chargé de vous présenter ses remerciements pour le don de votre livre et c'est une mission que j'ai acceptée avec grand plaisir. J'ai mis deux exemplaires en vente chez un libraire de mes amis.

J'ai reçu une lettre de Kennedy [?] à laquelle était jointe copie des propositions de Princeton aux Presses universitaires [de France]. Celles-ci ne m'ont parlé de rien et n'ont pas à le faire, étant propriétaires du livre. Si l'offre est acceptée, je pense comme vous que la tâche de Kennedy sera rude; mais puisque vous inclinez à lui faire confiance, tout est bien.

Nous avons en effet été fort surpris par le succès du Président Truman. Je pense que cet événement est favorable à la cause de la paix. Mais nous ne sommes pas tirés d'inquiétude. La confusion ne fait que croître.

Si vous en venez à une seconde édition de votre Coming of the French Revolution, je ne sais si vous pourrez y introduire une correction. J'ai découvert une autre bévue : je vous joins l'indication.

Veuillez bien agréer mes vœux pour votre famille et pour vousmême, et présenter mes hommages à Madame Palmer.

Bien amicalement vôtre 


\section{Lefebvre}

\section{[Appendice]}

Page $86,8^{\mathrm{e}}$ ligne (The Coming : p. $77: 3^{\mathrm{e}}$ ligne)

L'épisode auquel je fais allusion se rapporte au 23 juin et non au 5 mai. Il faudrait donc modifier le passage à peu près ainsi :

Le 5 mai, les trois ordres s'assemblèrent dans une salle récemment construite sur la rue des Chantiers, derrière l'hôtel des Menus-Plaisirs qui bordait la rue de Paris. Quand le roi se fut assis et couvert, les privilégiés se couvrirent aussi et le Tiers, contre l'usage, s'empressa de les imiter, ce que voyant, Louis XVI se découvrit de nouveau et tout le monde dût en faire autant.

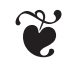

Boulogne-sur-Seine, 18 juin 1949

Cher ami,

J'ai tardé à vous répondre parce que j'ai négligé le courrier pour m'occuper constamment du Recueil des États-Généraux. Le tome ${ }^{\mathrm{er}}$ a dû être divisé en deux parties. La première a été enfin remise hier à l'Imprimerie nationale avec laquelle le Centre national de la recherche scientifique, qui a accepté de faire les frais de l'édition, a fini par traiter pour la publication. Pourparlers, lenteurs de leur associée, $M^{\text {lle }}$ Terroine, que le souci de l'érudition à ne jamais terminer à regret, $\mathrm{m}$ 'ont pris beaucoup de temps et procuré aussi des ennuis.

J'ai été surpris d'apprendre que l'édition de votre traduction était déjà épuisée. Voilà ce que c'est que de disposer du vaste public anglo-saxon. Je puis bien ajouter pourtant qu'on n'est vraiment jamais prophète en son pays, car très peu de mes compatriotes ont acheté mon livre, bien qu'il soit resté fort bon marché ; il est vrai que la vente a été confiée à une maison qui ne fait aucune publicité. Quoi qu'il en soit je souhaite bonne chance à la seconde édition.

Mais j'ai vu dans le Journal of Modern History que tous vos collègues ne sont pas contents que vous m'ayez traduit. Le critique estime que je n'ai rien dit qu'on ne sût déjà et que, d'autre part, l'Amérique ne manque pas d'historiens qui auraient fait, pour le moins aussi bien, et qui sont, en anglais, beaucoup plus brillants écrivains que moi, un français. Je n'ai rien à répondre et m'en rapporte à vous pour juger de ces opinions ${ }^{1}$. 
Mais ce qui m'a surtout intéressé, c'est que l'auteur, sans se découvrir nettement, me semble avoir été surtout indisposé en constatant qu'à mon avis, la Révolution française, telle qu'elle s'est faite le 4 août 1789, a dû son succès à l'insurrection populaire. Je conçois qu'on le regrette, mais non qu'on le conteste. Je sens fort bien dans ce grief l'arrière-pensée que nourrit la préoccupation contemporaine. Évidement, l'auteur a flairé le marxisme et je serais bien fâché - non d'être inscrit sur la liste des gens à qui on doit refuser l'entrée des États-Unis, car je suis bien âgé pour entreprendre le voyage - mais d'apprendre qu'on vous rend responsable de mes opinions.

Les dollars dont vous voulez bien me rappeler le souvenir commencent à faire une très grosse somme. N'hésitez pas à en disposer pour vous-même. Vous savez mon sentiment à l'égard de l'abandon généreux que vous avez tenu à faire à mon profit sur vos droits d'auteur. Je reste plein de scrupules vous voir vous en priver.

J'ai vu ici Mrs. Cameron [?] qui prépare un travail sur la peur en 1848. Je ne sais si vous la connaissez. Nous nous sommes entretenus de l'opinion américaine. Elle estime qu'en France, on s'exagère sa nervosité. Nous ne demandons qu'à nous rassurer car, ainsi que vous le dites, notre situation n'est pas précisément séduisante.

Mais d'un autre côté, on raconte ici également que la crise économique commence à se manifester chez vous. Par la baisse à la Bourse notamment. Comme je vous l'ai dit, c'est cette crise que je redoute principalement parce qu'on représenterait sans peine aux chômeurs que la guerre leur fournirait du travail.

Nous aussi commençons à voir grossir le nombre des chômeurs et ce n'est pas non plus pour améliorer nos perspectives.

Nous, historiens, ne pouvons qu'assister en spectateurs à l'évolution et travailler comme si elle devait prendre une tournure favorable.

Vous ne m'annoncez pas votre venue en France. Chinard m'a écrit qu'il s'embarquait. Il ajoute qu'on a commencé des recherches chez les Dupont de Nemours et qu'elles n'ont rien procuré jusqu'à présent.

Présentez mes hommages à Madame Palmer. Je suis persuadé que vos enfants continuent à grandir à votre satisfaction.

\section{Bien affectueusement}

\section{Lefebvre}

(1) Voir le compte rendu, assez sévère, par John Hall StewaRT, Journal of Modern History, t. 21, 1949, p. 60-62, de la traduction de R. R. PALmer, The Coming of the French Revolution, Princeton, Princeton University Press, 1947, 233 p. 
Boulogne s/S, 30 octobre [19]49

Cher ami,

Excusez la date tardive de cette réponse. Depuis la fin d'avril, j'ai pris froid à deux reprises, à Boulogne même d'abord, puis à Grasse où ma fille m'avait emmené pour m'en remettre. Quand nous sommes rentrés le 20 octobre, j'avais la bronchite et le médecin m'a consigné. Cela passera mais je ne sors pas encore. Le pire est la dépression qui a arrêté mon travail depuis deux mois. J'en étais arrivé au 18 brumaire dans la rédaction du nouveau volume de la collection Halphen et Sagnac ${ }^{1}$ et je me promettais de terminer au début d'octobre ! À présent, j'ai sur les bras les premières épreuves du Recueil des États-généraux et le manuscrit à mettre au point du premier volume des discours de Robespierre $^{2}$.

J'ai bien reçu le Spirit et votre compte-rendu ${ }^{3}$. Si vous êtes d'accord, je le traduirai pour le donner dans les Annales.

Votre étudiant ne m'a pas encore donné signe de vie et comme je ne possède pas son adresse à Paris, je ne sais comment le joindre. Je le mettrais volontiers avec mon collègue [Charles] Pouthas ${ }^{4}$ qui préside le Comité Tocqueville. Peut-être pourra-t-on arranger son affaire. Dites-lui de venir me voir.

J'ai bien reçu votre chèque de $\$ 100$. Votre générosité est inépuisable et je ne sais trop comment vous exprimer notre gratitude. Si vous êtes d'accord, je remettrai la somme à la Société des Études robespierristes en votre nom pour contribuer à l'édition des œuvres de Robespierre. Nous avons le manuscrit des Lettres aux Commettants établi par [Gustave] Laurent ${ }^{5}$ sans avoir encore trouvé les fonds pour l'impression. Je pense que cet emploi vous conviendra. Je me doute bien que le débat de Coming va se ralentir, mais le succès dépasse déjà mes espérances.

J'espère que l'hiver ne vous éprouvera pas. Agréez mes souhaits pour votre famille comme pour vous-même et croyez à mes affectueux sentiments.

\section{Lefebvre}

(1) La Révolution française, nouvelle édition du volume paru en 1930, Collection « Peuples et Civilisations », t. XIII, Paris, Presses universitaires de France, 1951, 674 p.

(2) Euvres de Maximilien Robespierre, t. 6, I ${ }^{\text {re }}$ partie, Discours, 1789-1790. Édition préparée sous la direction de Marc Bouloiseau, Georges Lefebvre et Albert Soboul, Paris, Presses universitaires de France, 1950, 703 p. 
(3) Cornwell B. Rogers, The Spirit of Revolution in 1789. A Study of Public Opinion as Revealed in Political Songs and Other Popular Literature at the Beginnings of the French Revolution, Princeton, Princeton University Press, 1949, 363 p. Compte rendu par G. LefEBvre, $A H R F$, t. 22, 1950, p. 391-392.

(4) Charles Pouthas (1886-1974), spécialiste de l'histoire française du XIX ${ }^{\mathrm{e}}$ siècle, professeur à la Sorbonne de 1930 à 1956. Il collaborait à diverses revues, dont les AHRF et la Revue d'histoire moderne et contemporaine.

(5) Gustave Laurent (1873-1949), historien rémois. Il préparait une édition critique des Lettres à ses Commettans de Robespierre, publiée à Gap, Imprimerie Louis-Jean en 1961. Sur la vie et l'œuvre de Laurent, voir l'article nécrologique paru dans les $A H R F$, t. 22, 1950, p. 184-192.

École Pratique

17, Rue de la Sorbonne (Odéon 24-13)

Des Hautes Études

$6^{\mathrm{e}}$ Section

Sciences Économiques et Sociales

Sorbonne

Cher ami,

Je suis content que la traduction italienne vous ait été de quelque intérêt. Il n'y a pas à s'étonner que les points de vue ne soient pas identiques, ni à le regretter car les différences approfondissent la connaissance. Pour ce qui est de Princeton, je me doute bien que ses vues dominent l'opinion chez vous, surtout en ce moment. J'aperçois ensuite dans votre lettre que la situation ne laisse pas de vous préoccuper et je pense qu'il y a de quoi. Tant que la crise économique est conjurée chez vous, on peut espérer qu'on évitera le pire. Mais les contribuables américains ne se fatigueront-ils pas de faire les frais du Plan Marshall et des fabrications de guerre ?

Vous attendez donc un troisième enfant. Je fais des vœux pour que sa naissance soit pleinement heureuse et pour que l'existence lui soit légère. Je ne pensais pas, je l'avoue, que la natalité s'accroisse chez vous comme ici. Un gonflement démographique ne va pas sans inconvénients éventuels. Si la production s'accroît en proportion, surtout du point de vue agricole, elle les parera. Mais il ne faudrait pas que la guerre s'empare du surplus. En attendant, je conçois qu'un voyage à destination de l'Europe vous soit interdit. 
Je vois que votre Coming continue à trouver des lecteurs et naturellement je m'en félicite. Je vous remercie de m'en avoir fait profiter en m'envoyant quelques volumes. Ils me sont bien arrivés mais je suis tellement pressé de divers côtés, d'achever différentes tâches que je n'ai encore pu les lire. Il est heureux que vous ayez pris connaissance du livre de [Jacques] Droz sur les Cisrhénans ${ }^{1}$. Vous trouverez dans les Annales un compte-rendu de celui qu'il a consacré à l'Allemagne au temps de la Révolution ${ }^{2}$. Il est encore plus important.

Je pense que vous avez vu ce temps dernier à Princeton, notre ancien étudiant, Chabert ${ }^{3}$, qui a pu séjourner quelques mois aux ÉtatsUnis grâce à une bourse Rockefeller. Ses thèses ${ }^{4}$ sont originales sur l'économie napoléonienne, mais sa situation me préoccupe. Il a 35 ans et c'est trop tard pour qu'il entre dans les cadres de notre enseignement qui, comme vous le savez, sont plus rigides que chez vous.

Son père avait enseigné en Roumanie et servi dans l'armée de ce pays, ce que lui a fait perdre la nationalité française. Chabert a été réintégré de droit sur sa demande, mais il a aussi perdu bien du temps. Êtesvous d'opinion qu'une chance se présenterait pour lui d'obtenir un poste dans une université américaine ? J'en doute.

Vous me rendriez service, à propos de mes bibliographies dans la nouvelle rédaction du volume sur la Révolution dans la collection Halphen et Sagnac, en m'indiquant quels sont les ouvrages que je dois indiquer sur l'histoire des États-Unis, aussi bien économique que politique, et notamment sur l'histoire sociale et culturelle. J'en connais plusieurs, mais je voudrais savoir quels sont ceux qui, aux yeux de vos collègues américains, paraissent les meilleurs.

Bien amicalement vôtre

\section{Lefebvre} $1940,72 \mathrm{p}$

(1) Jacques Droz (1909-1998), La pensée politique et morale des Cisrhénans, Paris, Sorlot,

(2) L'Allemagne et la Révolution française, Paris, Presses universitaires de France, 1949, 501 p. Voir le compte rendu par G. Lefebvre, $A H R F$, t. 22, 1950, p. 89-93.

(3) Alexandre Chabert, historien de l'économie française.

(4) Essai sur les mouvements des prix et des revenus en France de 1798 à 1820. [t. I], Paris, Librairie de Médicis, 1945, 271 p. Voir le compte rendu par G. Lefebvre, $A H R F$, t. 17, 1945, p. 83 ; Essai sur les mouvements des revenus et de l'activité économique en France de 1798 à 1820 [t. II], Paris : M.-T. Génin, 1949, 492 p. Compte rendu par G. Lefebvre, $A H R F$, t. 20, 1950, p. 285. 
Cher ami,

J'ai tardé à vous remercier de tous vos envois. En prévision de mon absence, des rédactions pressaient et l'approche du Congrès ${ }^{1}$ a multiplié les visiteurs. Je ne vous ai pourtant pas oublié, croyez-le, et me suis fait maints reproches. J'ai aussi reçu le volume I $^{\text {er }}$ des papiers de [Thomas] Jefferson. C'est une publication magnifique2. J'ai écrit un compte-rendu pour la Revue historique ; il passera également dans les Annales ${ }^{3}$. Puis-je vous demander de remercier, de ma part, $\mathrm{M}^{\mathrm{r}}$ Datus Smith.

J'espère que vous avez passé tranquillement cet été. Je présume que vous ne tarderez pas à posséder un nouvel héritier. Je fais des vœux pour sa maman en même temps que pour vous.

Ici, l'été a été maussade et orageux ; à présent, le temps est pluvieux et nous nous trouvons autant dire en automne : aujourd'hui, il fait frais. Je pars ce soir avec ma fille pour Grasse où je pense qu'il fera meilleur. Je rentrerai vers le 20. Ma fille qui dirige le personnel à l'hôpital de la Salpêtrière prend son congé qui est de trois semaines environ et a insisté pour que je lui tienne compagnie.

Le Congrès prendra fin demain. Je n'ai pu y assister que jeudi matin [31 août] et il m'a fallu y parler ${ }^{4}$. Les Américains se sont trouvés moins nombreux qu'on ne l'espérait et les circonstances l'expliquent ; les touristes sont repartis, dit-on, à la nouvelle que la situation internationale s'aggravait.

Depuis votre dernière lettre, elle a empiré en effet. Pour l'instant, il semble que votre gouvernement se montre conciliant en ce qui concerne Formose et qu'on pourra éviter une guerre entre la Chine et les ÉtatsUnis, laquelle compliquerait encore l'état du monde. Mais il me semble que l'on attend vos prochaines élections et que leur résultat aura de l'influence sur les péripéties futures. Comme vous pensez, les préoccupations ici sont vives et j'ai l'impression que l'opinion devient hésitante, quoique très hostile à la Russie.

Avez-vous vu l'article de Frances Acomb sur Albert Mathiez dans le volume publié à Chicago en 1942 par B. E. Schmitt sous le titre Some Historians of Modern Europe ${ }^{5}$ ? Si vous le jugez bon, vous pourriez m'envoyer sur lui quelques lignes de compte-rendu.

[Frederick John] Teggart a edité, paraît-il, en 1939, à Berkeley, un volume : Rome and China. A Study of Correlations in Historical Events ${ }^{6}$. Je suppose qu'il a voulu donner un exemple de l'orientation qu'il a recommandé de donner à l'histoire. 
J'ai vu aussi mention de R. Brunhouse, The Counter-Revolution in Pennsylvania, paru à Philadelphie en $1942^{7}$. Cela me paraît en rapport avec les publications de [Charles] Beard ${ }^{8}$ que j'ai trouvées à la Bibliothèque de la Sorbonne et dont j'ai fait bon usage. Vous me direz ce que vous pensez de tout cela.

Avec mes bons souhaits pour Madame Palmer, pour vos fils, pour vous (comme pour le monde entier !). Je vous envoie l'expression de mes sentiments affectueux.

\section{Lefebvre}

P. S. J'ai vu Gottschalk et Miss Hyslop qui vous parleront du Congrès quand vous aurez occasion de les voir.

(1) Le IX ${ }^{e}$ Congrès international des sciences historiques se réunissait à Paris du 28 août au 3 septembre 1950 . Press, 1950

(2) The Papers of Thomas Jefferson, Julian Boyd, éd., t.1, Princeton, Princeton University

(3) Signalé dans les $A H R F$, t. 23, 1951, p. 107.

(4) G. LefEBvre y présenta une communication sur Gracchus Babeuf. Voir Rapports, Paris, Librarie Armand Colin, 1950, p. 561-571.

(5) Frances Асомв, «Albert Mathiez (1874-1932)», dans Bernadotte E. Sснмiтt (éd.), Some Historians of Modern Europe, Chicago, University of Chicago Press, 1942, p. 306-323.

(6) Frederick J. Teggart, Rome and China : A Study of Correlations in Historical Events, Berkeley, University of California Press, 1939, 283 p.

(7) Robert L Brunhouse, Counter-Revolution in Pennsylvania, 1776-1790, Harrisburg, Pennsylvania Historical Commission, 1942, 368 p.

(8) Charles A. Beard (1874-1948), historien américain progressiste.

Université de paris Boulogne s/seine, 4-11-50

Faculté des lettres

Institut d'histoire

de la Révolution Française

Cher ami,

L'année scolaire a commencé pour nous, il y a longtemps déjà. J'espère qu'elle se poursuit pour vous dans de bonnes conditions. La situation générale est de plus en plus inquiétante, ce qui est malheureusement conforme aux prévisions ou du moins aux probabilités. 
Si la santé des vôtres ne vous procure pas d'inquiétudes et si vousmême pouvez travailler sans trop de difficultés, j'ai lieu de croire que vous prenez le parti d'attendre avec calme.

J'ai bien reçu vos envois. J'admire que vous ayez pu mettre sur pied cette histoire de l'Europe moderne ${ }^{1}$; sans avoir pu encore la lire de bout en bout, j'ai pris connaissance de bon nombre de parties et des bibliographies. Votre récit présente la même indépendance que celui des Twelve Who Ruled ${ }^{2}$ et il conserve bien l'allure alerte et panoramique de la synthèse. J'ai aussi tiré profit des indications bibliographiques de Commager ${ }^{3}$, complétées parfois par vous puisqu'elles ont le tort, qui est grave, [de] ne pas dater les ouvrages. Je vous remercie de m'avoir également éclairé sur quelques-uns des ouvrages relatifs aux États-Unis qui étaient cités jusqu'à présent dans le volume sur la Révolution.

Je vais de nouveau recourir à vous concernant quelques autres. Dans mon volume sur Napoléon ${ }^{4}$, j'ai cité Thurman W. Van Metre, Economic History of U.S.A., paru à Londres, 1925․ Connaissez-vous l'ouvrage et ne faut-il pas l'éliminer ? Commager, en effet, ne le cite pas. Peut-être serez-vous d'avis de lui substituer soit C. W. Wright, Economic History of the U. S. ${ }^{6}$, soit H. U. Faulkner, American Economic History ${ }^{7}$, soit l'un et l'autre ; Commager les indique tous deux, mais je ne les trouve pas ici. Et en outre, comme je l'ai rappelé, Commager n'indique ni lieux ni dates.

Voici, pour finir, un cas plus embarrassant encore. J'ai trouvé dans les listes d'ouvrages parus que produit The Journal of Modern History (1949, p. 143), l'indication suivante :

Holden Furber, John Company at Work: A Study of European Expansion in the Late Eighteenth-Century, Harvard Historical Studies, t. LV, 1948, 407 pages.

Un pareil titre a éveillé vivement ma curiosité. Le livre doit viser les compagnies anglaises et hollandaises principalement, car la France n'a guère à aligner que la Compagnie des Indes. Malheureusement, la Sorbonne n'a pas reçu les Harvard Studies depuis 1939 et les bibliothèques américaines de Paris ne les ont pas non plus, comme trop spéciales. Puis-je vous demander si le livre de Furber vous paraît digne de considération? Et dans l'affirmative, pourriez-vous m'en envoyer un exemplaire?

Je suis d'ailleurs un peu perplexe sur les Harvard Studies. D'après le Journal of Modern History, le livre de Furber fait partie des Harvard Historical Studies ; or, le livre de Harris, The Assignats, porte la mention : Harvard Economic Studies ( ${ }^{\circ} 33$ ) ; je suppose que c'est la même collection? 
Je m'excuse de vous procurer tant de tracas supplémentaire et je vous remercie pour votre inépuisable obligeance.

J'ai vu [Louis] Gottschalk ${ }^{8}$ cet été ; nous nous sommes aussi rencontrés au Congrès avec [Charles K.] Webster ${ }^{9}$, [R. H.] Tawney ${ }^{10}$ et [Alfred] Cobban ${ }^{11}$ notamment. Il aurait été bien agréable de vous y voir ainsi que [Leo] Gershoy ${ }^{12}$. Miss [Beatrice] Hyslop ${ }^{13}$ était venue. Espérons que l'avenir nous réunira.

\section{Bien amicalement vôtre}

\section{Lefebvre}

(1) A History of the Modern World, New York, Knopf, New York, 1950, 900 p.

(2) Twelve Who Ruled: The Year of the Terror in the French Revolution, Princeton, Princeton University Press, 1941, $417 \mathrm{p}$.

(3) Henry S. Commager (1902-1998) et Samuel Eliot Morison (1887-1976), $4^{e}$ éd., 2 vol., The Growth of the American Republic, New York, Oxford University Press, 1950, 895 p.

(4) Napoléon, Collection «Peuples et Civilisations », t. XIV, Paris, F. Alcan, 1935, 606 p.

(5) Thurman W. Van Metre (1884-1961), Economic History of the United States, Londres, Pitman, 1925, $672 \mathrm{p}$.

(6) Chester W. Wright (1879-1966), Economic History of the United States, 2e éd., New York, McGraw-Hill, 1949, 441 p.

(7) Harold U. FaulKner (1890-1968), American Economic History, 6 e ed., New York, Harper, 1949.

(8) Louis Gottschalk (1888-1975), historien américain, spécialiste de l'histoire révolutionnaire.

(9) Charles K. Webster (1886-1961), historien britannique, spécialiste de l'histoire diplomatique. et sociale.

(10) R. H. Tawney (1880-1962), historien britannique, spécialiste de l'histoire économique

(11) Alfred Cobban (1901-1968), historien britannique, spécialiste de l'histoire française.

(12) Leo Gershoy (1891-1975), historien américain, spécialiste de l'histoire révolutionnaire.

(13) Beatrice Hyslop (1899-1973), historienne américaine, spécialiste des cahiers de doléances de 1789 . 
Université de Paris

Boulogne s/S, 3-9-51

Faculté des Lettre

Institut d'Histoire

de la Révolution Française

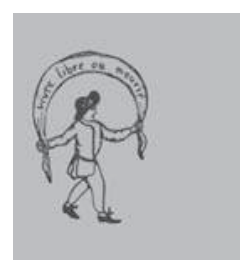

Cher ami,

Je me suis senti un peu ému en recevant votre pli parce que l'enveloppe me portait à me demander si vous aviez quitté Princeton. Votre lettre m'a rassuré. Nous ne sommes pas sans avoir entendu parler du Colorado ${ }^{1}$. Je crois me souvenir de lettres échangées avec un professeur de Boulder, sans parvenir à retrouver son nom, et mon ami et ancien étudiant [Charles] Morazé est allé donner des conférences à Denver c'est-à-dire probablement à Boulder.

Je vous remercie d'avoir répondu à mes questions. Pour ce qui est de [Daniel] Guérin ${ }^{3}$, il a récolté de même ici des signatures pour une pétition à votre gouvernement, afin d'être de nouveau autorisé à entrer chez vous. Il y déclare qu'il n'est pas communiste et il est bien certain qu'il me paraît plutôt anarchiste libertaire. Dans le livre que vous savez ${ }^{4}$, il faut toujours, pour le bien comprendre, lire Staline quand il écrit Robespierre. Naturellement, il ne s'est pas adressé à moi et je n'aurais pas conseillé de plaider sa cause avant qu'il ne m'explique pourquoi il a installé sa femme et sa fille aux États-Unis. Serait-ce pour les mettre à l'abri lorsque la guerre aura éclaté ? Je n'insiste pas sur ce dernier sujet. Il y aurait trop à dire et je préfère attendre que nous puissions nous en entretenir de vive voix. J'espère toujours que vous réussirez sans trop attendre à faire le voyage ; n'en déduisez pas que je n'aie point égard à la difficulté. Il y a un demi-siècle, les charges familiales m'ont procuré bien de tourments. La marche des événements augmente chaque jour mon inquiétude. Il y a aussi chez nous des gens qui aspirent à une guerre de croisade contre les Soviets et [Raymond] Aron, dont vous citez le nom, ne fait pas mystère de son opinion : il faut détruire le «monstre », écrit-il. La contradiction que vous signalez chez ceux qui veulent armer a outrance, et en même temps, ne pas diminuer le niveau de vie, est beaucoup plus sensible encore dans un pays ruiné comme la France qu'elle ne peut l'être chez vous. La contradiction que vous signalez par suite devient internationale parce que, comme je l'avais prévu, la France s'étant condamnée à l'inflation vertigineuse, par le réarmement et la guerre en Indochine, va se retourner ver les États-Unis en lui demandant de fournir l'argent nécessaire, faute de quoi, elle s'effondrera. Or, il ne me semble pas qu'à l'approche de nos élections, le Congrès soit disposé à répondre favorablement. 
Je suis content d'apprendre que l'on traduit le dernier livre de Marc Bloch $^{5}$. Malheureusement, il est inachevé et [Lucien] Febvre ${ }^{6}$ qui savait, en partie au moins, ce que Bloch projetait de dire en sus, n'a pas jugé à propos de s'en expliquer. Aussi le livre ne contient-il guère que ce que nous admettons aujourd'hui, pour la plupart, sans discussion. J'ai signalé dans un article de la Revue historique (Avenir de 1'histoire) ${ }^{7}$ en 1947, à propos d'un livre d'Halphen, un passage de Marc Bloch, tiré d'un volume qui n'est sans doute pas connu aux États-Unis (L'Étrange défaite) ${ }^{8}$, passage qui laisse entrevoir ce qu'aurait contenu le chapitre qu'il n'a pas eu le temps d'écrire pour « Le Métier d'historien ».

Les notions d'historiographie moderne dont vous avez trouvé mention sont un cours que j'ai donné à la Sorbonne la dernière année de mon enseignement (1944-1945) et qui a été ronéotypé ${ }^{9}$. Je vous en ai envoyé un exemplaire. J'avais signalé à mes collègues qu'il était déplorable qu'un étudiant sortît de la Sorbonne sans avoir aucune idée du développement de sa discipline et sans rien connaître que le nom (et encore !) des grands historiens français et étrangers. J'ai essayé de combler la lacune fort imparfaitement. Vous le constaterez car je n'ai pas parlé de l'historiographie américaine que je connais fort incomplètement. Depuis, j'ai continué à ruminer le sujet et, d'autre part, on m'a demandé d'écrire un livre à son propos. Pour l'une et l'autre raison, je m'y déciderai peut-être ; mais aurai-je le temps ? Ce ne serait pas inutile, car, après mon départ, mes collègues n'ont eu aucun égard à mon essai et aucun ne l'a poursuivi.

Le volume de [Jacques] Godechot ${ }^{10}$ est en effet considérable. Il ne me satisfait pas entièrement, vous le verrez prochainement dans les Annales ${ }^{11}$. Tel quel, je pense qu'il a été écrit trop vite et sans un délai de méditation suffisant.

Harvard m'a envoyé le dernier livre de [Donald] Greer ${ }^{12}$. Je suis d'accord avec vous sur les mérites de sa méthode et de l'effort qu'il a dû s'imposer. Il est souhaitable que nos étudiants connaissent ses travaux et ceux d[e] [Seymour] Harris ${ }^{13}$. Ils font grand honneur aux États-Unis.

Le premier volume des États-généraux reste à l'Imprimerie nationale. Ma collaboratrice, $\mathrm{M}^{\text {lle }}$ Terroine, a de graves difficultés de santé. Par suite, quand elle a eu le manuscrit entre les mains, elle ne l'a pas ouvert et, pourtant, elle m'a laissé le porter à l'Imprimerie sans m'en prévenir ; puis, quand les épreuves sont arrivées, elle a multiplié les observations et elle s'occupe encore à les bouleverser. Tout reste donc en désordre et je ne sais même pas encore si le Centre [national] de la Recherche [scientifique] qui fait les frais de l'édition ne va pas s'effrayer du prix des corrections. 
Les discours de Robespierre suivent plus normalement leur cours. On imprime le second volume ${ }^{14}$ et les autres sont à peu près préparés.

Les Presses universitaires [de France] mettront en vente en octobre ou novembre ma nouvelle rédaction du tome XIII consacré à la Révolution dans la collection Halphen et Sagnac. Je vous l'enverrai.

Elles vont aussi réimprimer Napoléon qui est à peu près épuisé ${ }^{15}$. J'ai fait la toilette du volume et mis à jour les bibliographies.

Je ne puis qu'approuver votre projet en principe. Il vous demandera beaucoup de lectures et la réalisation de la synthèse ne sera pas facile. Nous en reparlerons quand votre plan se sera précisé. Mais c'est une synthèse bien propre à vous tenter ${ }^{16}$.

Vous ne me parlez pas de votre petite fille [Emily] : j'espère qu'elle grandit paisiblement. Vos fils doivent commencer à intéresser vivement un éducateur. Mais quelle tâche pour leur maman et leur papa! Je n'ai élevé que deux filles. Ainsi je mesure vos préoccupations !

Je vous souhaite bon courage et bonne santé. Croyez à mes sentiments de cordial attachement.

\section{Lefebvre}

(1) En effet, R. R. PALmer enseignait à l'Université de Colorado à Boulder pendant l'été de 1951.

(2) Charles Morazé (1913-2003), historien et homme politique, co-directeur des Annales Économies, Sociétés, Civilisations et créateur de l'École des Hautes études en sciences sociales.

(3) Daniel Guérin (1904-1988), marxiste de tendance trotskiste et anticolonialiste. Résidant aux États-Unis de 1946 à 1949 lorsqu'il fut expulsé.

(4) La Lutte de classes sous la première République : Bourgeois et « bras nus » (1794-1797), 2 vol., Paris, Gallimard, 1946. Voir le compte rendu par G. LefEBVRE, AHRF, t. 19, 1947, p. 173-179.

(5) Marc Bцoch (1886-1944), Apologie pour l'histoire ou métier d'historien, Paris, A. Colin, 1949, 112 p. Traduction anglaise par Peter Putnam, Historian's Craft, New York, Knopf, 1953, $197 \mathrm{p}$.

(6) Lucien Febvre (1878-1956), co-fondateur, avec Marc Bloch, des Annales d'histoire économique et sociale.

(7) G. LeFEBvre, « L'Avenir de l'histoire », Revue historique, t. 192, 1947, p. 55-61, compte rendu par L. Halphen, Introduction à l'histoire, Paris, Presses universitaires de France, 1946, 99 p.

(8) Marc Bloch, L'Étrange défaite, Paris, Éditions Franc-tireur, 1946, 194 p. Traduction anglaise par Gerard Hopkins, Strange Defeat, Londres et New York, Oxford University Press, 1949, $178 \mathrm{p}$.

(9) G. Lefebvre, Notions d'historiographie moderne, Cours de la Sorbonne, Paris, Centre de documentation universitaire, 1946 ; nouvelle édition : La Naissance de l'historiographie moderne, Collection « Nouvelle bibliothèque scientifique », Paris, Flammarion, 1971, 349 p.

(10) Jacques Godechot, Les Institutions de la France sous la Révolution et Napoléon, Collection « Histoire des institutions », Paris, Presses universitaires de France, 1951, 688 p.

(11) Compte rendu par G. LeFEBvre, AHRF, t. 23, 1951, p. 416-422.

(12) Donald GREER (1896-1978), The Incidence of the Emigration during the French Revolution, Harvard Historical Monographs, n²4, Cambridge, Mass., Harvard University Press, 1951, 173 p. Compte rendu par G. Lefebvre, $A H R F$, t. 23, 1951, p. 416-422. Greer avait publié auparavant 
The Incidence of the Terror during the French Revolution, Harvard Historical Monographs, n 8 , Cambridge, Mass., Harvard University Press, 1935, 196 p. Compte rendu par G. Lefebvre, AHRF, t. 14,1937 , p. $164-166$.

(13) Seymour E. Harris (1897-1974), The Assignats, Harvard Economic Studies, n 33 , Cambridge, Mass., Harvard University Press, 1930, 293 p. Compte rendu par G. Lefebvre, Annales d'histoire économique et sociale, t. 3, 1931, p. 628-630.

(14) Euvres de Maximilien Robespierre, t. 7, Discours, 2e partie, janvier-septembre 1791. Édition préparée sous la direction de Marc Bouloiseau, Georges Lefebvre et Albert Soboul, Paris, Presses universitaires de France, 1951, 784 p.

(15) Napoléon, $3^{e}$ édition, Collection « Peuples et Civilisations », t. XIV, Paris, Presses universitaires de France, 1951, 220 p.

(16) Il s'agit sans doute de sa thèse sur la «Révolution atlantique », lancée avec Jacques Godechot lors du $\mathrm{X}^{\mathrm{e}}$ Congrès international des sciences historiques réuni à Rome en août 1955 et mise à point dans son étude The Age of the Democratic Revolution, 2 vol., Princeton, Princeton University Press, 1959-1964. Voir J. Godechot, « Le X ${ }^{\mathrm{e}}$ Congrès international des sciences historiques », $A H R F$, t. 28,1956, p. $104-105$.

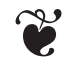

Boulogne-sur-Seine, 26 décembre 1951

Cher ami,

Je déplore que l'hiver vous ait déjà éprouvés. En vous exprimant mes remerciements pour vos souhaits, je m'empresse de former des vœux pour que l'amélioration que vous m'annoncez se confirme. Ici, l'automne a été jusqu'à présent d'une douceur humide inusitée. Heureusement, car le charbon n'abonde pas. Ce temps n'est pas aussi favorable à la santé qu'on espérait et, en novembre, j'ai souffert d'une dépression qui a interrompu mon travail durant plusieurs semaines. C'est passé. Je viens d'achever une préface pour la réédition de l'Ancien régime et la Révolution de Tocqueville ${ }^{1}$. Sans doute, avez-vous appris que deux éditeurs ont réimprimé ici la Démocratie en Amérique.

J'ai des excuses et des regrets à ajouter en ce qui concerne ma Révolution. Je ne vous savais pas en rapport étroit avec le Journal of Modern History et je croyais Gottschalk très lié avec lui. Comme le nombre d'exemplaires dont je disposais était limité, je lui écrivis pour le prévenir que je gardais celui à lui destiné, mais que j'attendais pour le lui envoyer de savoir si on ne lui en avait pas confié le compte rendu, car, sur ma demande, les Presses universitaires [de France] avaient expédié à Chicago un exemplaire de presse $^{2}$.

Une lettre de M. Carson [?] me donnait à supposer qu'il vous avait précisément demandé ce compte-rendu. S'il en est ainsi, veuillez me 
pardonner d'avoir malheureusement paru contrarier l'arrangement. Il va de soi que, mieux informé, je me serais félicité de connaître votre avis.

À présent, je vais m'atteler à une révision de mon volume sur Napoléon; il est épuisé. Peut-être qu'il me sera permis de revenir à mes études sur la région orléanaise ${ }^{3}$.

Je regarde à nouveau la photographie de vos trois enfants. Ils me semblent prospères. L'harmonie règne visiblement entre eux et la benjamine a complété bien heureusement la trinité.

Bien affectueusement vôtre

\section{Lefebvre}

(1) Alexis de Tocqueville, Euvres complètes, J.-P. Mayer, éd., t. 2, L'Ancien Régime et la Révolution, 1, Paris, Gallimard, 1952, préface de G. LeFEBVRE, p. 9-30.

(2) Compte rendu par Leo Gershoy de G. Lefebvre, La Révolution française, nouvelle édition, Collection « Peuples et Civilisations », t. XIII, Paris, Presses universitaires de France, 1951, 674 p., Journal of Modern History, t. 25, 1953, p. 301-303.

(3) Ouvrage posthume: Études orléanaises, 2 vol., Paris, Bibliothèque nationale, 19621963, Commission d'histoire économique et sociale de la Révolution, Mémoires et documents, XV, t. 1, Contribution à l'étude des structures sociales à la fin du XVIII siècle, 276 p. ; t. 2, Subsistances et Maximum (1789-an IV), $476 \mathrm{p}$.

Boulogne-sur-Seine, 25-12-52

Cher ami,

Merci pour votre bonne lettre et pour vos vœux. Recevez mes souhaits pour vous et pour les vôtres. Je souhaite particulièrement pour ce qui concerne vos travaux que le temps vous soit donné de préparer le grand ouvrage dont vous m'avez fait connaître le projet.

Je vous remercie encore de m'avoir envoyé le petit livre de Stern [?] qui est de lecture facile et intéressante. Il ne révèle pas seulement le point de vue américain, mais aussi celui de beaucoup d'intellectuels allemands sans aucune doute. Je n'entreprendrai pas de le discuter. Vous avez fait en terminant des réserves et, fort sagement, vous vous êtes gardé d'insister. Le temps ne me paraît pas bien propre à contester aux ÉtatsUnis les vues si orthodoxes de l'auteur.

Je me contente de relever la première phrase du second paragraphe de la page 28 où il cite Marx ne laissant supposer qu'il définit les capita- 
listes comme une classe « strictement fonctionnelle ». Il est incroyable qu'il ne remarque nulle part que la propriété des moyens de production confère un monopole de ces fonctions - et qui plus est, un monopole en principe héréditaire, en sorte ce qu'il dit plus loin sur l'égale « opportunity » ne tient pas debout. Il aurait bien fait de nous dire combien il y a de fils d'ouvriers à Harvard ou à Columbia. Dans nos universités, nous n'en comptons pas 1 pour cent. Tout ce qu'il dit sur la supériorité de l'ordre capitaliste sur l'ordre féodal va de soi et la bienfaisance historique du capitalisme est notoire. Mais bien qu'il reste bon de le dire, cela ne clôt pas l'histoire.

La situation ici est d'une confusion inexprimable et l'inquiétude devient générale quant à la guerre d'Indochine et le réarmement de l'Allemagne, sans parler de la situation financière! La situation internationale reste dans le même état qui n'est pas rassurant. Nous attendons avec anxiété les décisions d'Eisenhower.

Bonne santé et bon courage !

Bien amicalement,

Lefebvre

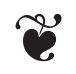

Boulogne-sur-Seine, 22 juin 1953

Cher ami,

J'ai tardé à vous écrire parce que ma fille aînée était gravement malade. Elle souffrait depuis longtemps d'un rétrécissement de la valvule mitrale et une endocardite infectieuse s'était déclarée qui ne laissait pas d'espoir. Je relayais ma cadette pour la veiller nuit et jour et mon travail s'était complètement interrompu. Elle est morte le dimanche 14 juin. Vous estimerez sûrement comme moi que tout commentaire serait superflu.

Je vous remercie de m'avoir envoyé cette note sur Melville ${ }^{1}$. Je la ferai passer dès que je pourrai. Il n'y aucun inconvénient à son insertion dans une revue américaine. Il est très bon, d'autre part, que Mirkine [Guetzévitch] ${ }^{2}$ publie en français vos excellentes réflexions.

Je ne compte pas quitter Boulogne désormais. Ainsi pourrais-je voir votre étudiant Stanley Mellon ${ }^{3}$. Mais il me semble qu'il aurait plus d'intérêt encore à entrer en rapport avec mon collègue Pouthas, de la Sorbonne, qui s'est spécialisé dans la période $1815-1848$ et se présente à 
nous comme le biographe de Guizot. Mellon pourrait lui écrire. Pouthas habite 32, avenue Rapp, Paris $\left(7^{\mathrm{e}}\right)$.

Celui de vos étudiants qui s'intéresse à l'histoire du protestantisme pendant la Révolution fera bien de se mettre en rapport avec [ÉmileGuillaume] Léonard ${ }^{4}$ qui est actuellement à l'École des Hautes études. Son adresse est : 1 Rue de la Porte jaune, Garches (Seine-et-Oise).

Je comprends bien les raisons qui vous empêchent d'entreprendre un nouveau voyage vers l'Europe et la France. Vous savez que je regrette ne pouvoir entrer directement en conversation avec vous. J'espère du moins que vos projets de publications pourront être menés à bien.

Quant à la traduction de mon livre [sur Napoléon], toutes les interventions y compris la mienne auprès des Presses universitaires [de France], semblent être demeurées vaines. Je ne puis dire pourquoi leurs vues essentiellement commerciales se sont opposées aux concessions demandées par Knopf ( $\mathrm{M}^{\mathrm{me}}$ [Blanche]). $\operatorname{Knopf}^{5}$ était venue me voir avec $\mathrm{M}^{\mathrm{r}}$ Bradley [?]. Cependant les traductions en allemand, en espagnol et en italien ont été autorisées. Je regrette amèrement que le monde anglo-saxon me soit ainsi partiellement fermé. Pour l'Allemagne et l'Espagne, la traduction de Napoléon a même été permise aussi.

Pour ce qui est d'un professeur français qui se rendrait à l'invitation de Princeton, l'invitation pourrait peut-être séduire [Jacques] Godechot, de Toulouse ${ }^{6}$; [Yves] Renouard, de Bordeaux ${ }^{7}$; [André] Fugier, de Lyon ${ }^{8}$. Je vais leur en écrire, mais j'ignore s'ils sont en état de parler anglais. Je vous ferai connaître leurs réponses.

Oui, j'entrerai dans la $80^{\mathrm{e}}$ année, le 5 août prochain. Je vous remercie de m'en féliciter. Je vais essayer de me remettre au travail.

Bien cordialement vôtre

\section{Lefebvre}

(1) R. R. PALMER, « Herman Melville et la Révolution française », $A H R F$, t. 26, 1954, p. 254-256.

(2) Boris Mirkine-Guetzévitch (1892-1955), spécialiste du droit international et constitutionnel.

(3) Stanley Mellon (1927-2008), historien américain, spécialiste de l'historiographie française du XIX ${ }^{\mathrm{e}}$ siècle.

(4) Émile-Guillaume Léonard (1891-1961), historien du Protestantisme français.

(5) Blanche Wolf Knopf (1893-1966), éditrice, femme d'Alfred A. Knopf.

(6) Jacques Godeснот (1907-1989), historien de la Révolution française, professeur et doyen de la Faculté des lettres de l'Université de Toulouse. C'est lui qui fut enfin désigné pour enseigner à

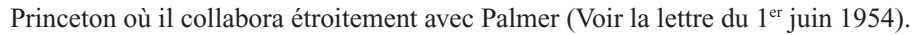

(7) Yves Renouard (1908-1965), médiéviste, professeur à l'Université de Bordeaux. sité de Lyon.

(8) André Fugier (1896-1976), historien de l'époque napoléonienne, professeur à l'Univer- 
Cher collègue,

Il y a bien longtemps que je ne vous ai donné mes nouvelles. La raison en est qu'en janvier, je suis devenu gravement malade. D'après le médecin, mes organes, malgré l'âge, fonctionnent encore de manière très satisfaisante, mais j'ai souffert d'une « dépression » vitale très prononcée. Qu'est-ce au juste que cette dépression? Il n'en sait rien et il paraît que les biologistes ne sont pas mieux renseignés. Il en rapportait l'origine à la mort de ma fille aînée, survenue le 14 juin dernier, et aussi au surmenage. Je crois que ses vues étaient justes. Quant au remède, il ne pouvait être qu'empirique puisque les modalités biologiques demeurent inconnues. Il m'a donc condamné au repos absolu au lit: ni visites, ni lectures, ni courrier. Ajoutez des drogues et des piqûres variées. Ce régime a duré trois mois. Enfin, en avril, un mieux s'est esquissé et peu à peu la vie normale a repris. Finalement, en mai, le médecin m'a invité à « changer d'air ». Je me trouve donc depuis quelques jours dans une maison de repos installée par les enseignants du Gard dans le domaine de Figaret, à St-Hippolyte-du-Fort, au nord de Nîmes. J'y resterai jusqu'au 25 juin. À part cela, j'ai peu de choses à vous conter. Ma société a tenue son assemblée générale le 15 mai. On y a prononcé quelques paroles à propos de mon entrée prochaine dans la quatre-vingtième année ${ }^{1}$.

L'assistance s'est montrée sensible à l'adresse que Miss [Beatrice] Hyslop lui avait envoyée de la part des historiens américains à propos de cet anniversaire ${ }^{2}$. Il n'est pas besoin d'ajouter que mon élection comme honoraire de l'Association des historiens des États-Unis a renforcé les sentiments de gratitude que nous éprouvions. Mes amis ont pris l'initiative de réunir en un volume des articles souvent anciens, éparpillés en diverses revues. Ces «Études sur la Révolution française » seront distribuées aux souscripteurs dans le courant de ce mois.

J'ai trouvé remarquables les articles que vous m'avez envoyés. J'y ai reconnu que vos travaux mènent leur cours et que vous n'avez pas abandonné le grand projet dont vous m'avez parlé.

[Jacques] Godechot m'a informé, d'autre part, qu'un accord avait été conclu entre Princeton et lui, et qu'il se trouverait cet hiver auprès de vous. Je veux espérer qu'il vous contentera.

Je n'ajoute rien sur la situation internationale et sur celle de mon pays. Si ce n'est toutefois qu'aux récentes nouvelles, la crainte d'une crise économique chez vous semble se dissiper et j'en suis heureux car une 
telle crise, comme je vous l'ai dit il y a longtemps déjà, me paraît l'éventuel facteur d'une poussée irrésistible vers une guerre préventive.

Je souhaite des vacances paisibles et en parfaite santé à tous les vôtres et à vous-même.

À vous bien fidèlement

\section{Lefebvre}

(1) « Un anniversaire », $A H R F$, t. 26, 1954, p. 94

(2) American Historical Review, t. 59, avril 1954, p. 819-820.

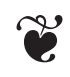

Boulogne s/S, 5-1-55

Cher ami,

Je vous remercie d'avoir pensé à m'envoyer un Christmas [sic], orné de la photographie familiale, et de lui avoir adjoint vos vœux. Veuillez bien agréer les miens pour vous et tous les vôtres.

J'ai lieu d'espérer que l'hiver ne vous a pas éprouvés. L'automne ici fut humide et plus doux que d'habitude. Jusqu'à présent, je n'en ai pas souffert. Mais je ne suis qu'incomplètement remis de la crise de l'hiver dernier, en ce que le travail me fatigue encore bien vite.

J'accueille avec un vif plaisir la nouvelle qu'en route pour le Congrès [international des sciences historiques] de Rome, vous comptez passer par Paris. Nous ne nous sommes jamais rencontrés. Quelle bonne occasion!

Bien affectueusement

Lefebvre 
Boulogne-sur-Seine, 6 février 1956

Cher ami,

C'est bien tardivement que je vous remercie de votre bon souvenir de Christmas et de vos vœux pour la présente année. Veuillez bien accueillir favorablement mes souhaits pour vous et pour les vôtres.

Ma correspondance s'est trouvée arrêtée par divers accrocs : une chute dans mon escalier fraîchement encaustiqué, puis un abcès dentaire fort pénible. Mon travail fut suspendu longuement; il reprend seulement.

J'ai été surpris de la nouvelle que vous m'envoyez des intentions d'[Alfred ] Knopf. Comme vous dites, c'est un triomphe - et une confirmation du proverbe d'après lequel on n'est jamais prophète dans son pays. Car mon Quatre-vingt-neuf est beaucoup moins connu en France que dans les pays de langue anglaise - et grâce à vous, cher ami. C'est une nouvelle occasion de vous témoigner ma fidèle reconnaissance.

Bien affectueusement

Lefebvre

Boulogne-sur-Seine, 15-5-56

Cher ami,

J'aurais dû vous écrire depuis longtemps pour vous remercier de m'avoir envoyé ces volumes. Mais j'espérais trouver le temps de les feuilleter, sinon de les lire ; principalement celui dont vous me dites qu'il est défavorable à [Charles A.] Beard dont les ouvrages comptent à mes yeux parmi les plus remarquables qui me soient parvenus de l'historiographie américaine ${ }^{1}$.

Il m'a fallu renoncer : j'ai trop à faire pour que le loisir soit durable ; c'est pourquoi j'ai pris la plume à votre adresse.

Bien entendu j'ai été enchanté d'apprendre que votre Coming a pris un nouvel essor ; puisqu'il paraît que c'est un paper-book [sic], je le présume à un prix plus modeste qui sera accessible à de nouveaux et nombreux lecteurs. 
Rien de nouveau en ce qui me concerne. Vous savez dans quel pétrin des gouvernants ineptes ont plongé mon malheureux pays. Nous n'inaugurons rien de bon de cette guerre d'Algérie ni pour la situation internationale de la France, ni pour le régime républicain. Qu'y faire ? Qui vivra verra!

Voici le printemps venu. J'espère que l'hiver ne vous a pas éprouvés, vous et les vôtres. Il ne m'a pas bien traité mais, pour le moment, c'est fini.

Bien affectueusement,

Lefebvre

(1) Il s'agit du livre de Robert E. BRown, Charles Beard and the Constitution, a Critical Analysis of «An Economic Interpretation of the Constitution », Princeton, Princeton University Press, 1956, 219 p., signalé dans les $A H R F$, t. 29, 1957, p. 90-91.

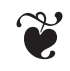

Boulogne-sur-Seine, 9 mars 1957

Cher ami,

Heureux que mon article sur la conférence d'[Alfred] Cobban ait obtenu votre assentiment ${ }^{1}$. La tendance que vous signalez ne s'explique que trop bien par la crainte sociale. [Louis] Gottschalk vient de le signaler (implicitement, car il montre beaucoup de discrétion dans ses critiques) à propos de Burke ${ }^{2}$.

Mais vous ne m'aviez pas encore communiqué le plan de votre ouvrage. Pour l'instant, je ne vois aucune réflexion à vous présenter. Je pense que le début de la guerre est en effet un tournant qui justifiera parfaitement la séparation entre les deux volumes.

Peut-être l'ouvrage de [Jacques] Godechot vous est-il parvenu. J'en dirai quelques mots prochainement dans les Annales ${ }^{3}$.

Je viens de recevoir deux exemplaires de l'édition publiée par Vintage Books à New York ${ }^{4}$. Vous me l'aviez annoncée. C'est une chance pour moi de me voir mis en contact enfin avec le public anglo-saxon. Je n'en peux dire autant du public français : on n'est jamais prophète dans son pays !

Nous sommes occupés à mettre en train l'enquête sur la structure sociale. Un colloque national a eu lieu dimanche [le 3 mars]. Il est possible 
que ce que j'ai dit soit réimprimé dans les Annales 5 . Je joins la circulaire qui avait été envoyée comme invitation.

D'après une lettre de Miss [Beatrice] Hyslop, cette enquête, décidée au Congrès [international des sciences historiques] à Rome, a fait l'objet d'une discussion à la récente réunion des historiens américains. Comme je m'y attendais, elle rencontre des opposants. Pourquoi ? Nous nous proposons de compter l'effectif et approximativement le revenu des diverses catégories sociales. Ceux qui ont parlé le plus franchement laissaient entendre que ce sont des faits qu'il vaut mieux laisser dans l'ombre. On retrouve ici la crainte sociale. Mais le rôle de l'histoire est de rechercher le fait. Le physicien qui étudie le phénomène nucléaire n'a pas à demander si ses découvertes procureront des bombes !

Notre intention est de convoquer, sans trop tarder, un colloque international pour éclairer l'existence des sources dans les différents pays. Mais nous nous sommes exposés fâcheusement à des délais car [Ernest] Labrousse $^{6}$ doit être opéré prochainement de la prostate et [Marc] Bouloi$\mathrm{seau}^{7}$ le sera la semaine prochaine de la vésicule biliaire.

Jusqu'à présent, je reste indemne.

\section{Bien affectueusement}

\section{Lefebvre}

(1) G. Lefebvre, «Le Mythe de la Révolution française », AHRF, t. 28, 1956, p. 65-71. Critique sévère de la thèse "révisioniste » d'Alfred Cobban, The Myth of the French Revolution, Londres, University College, 1955, 25 p.

(2) Louis Gottschalk, «Reflections on Burke's Reflections on the French Revolution », Philadelphie, Proceedings of the American Philosophical Society, t. 100, n 5, 1956, p. 417-429. Signalé dans les $A H R F$, t. 29, 1957, p. 288.

(3) Jacques Godechot, La Grande nation: L'Expansion révolutionnaire dans le monde, 1789-1799, Paris, Éditions Montaigne, 1956, 2 vol., 785 p. Compte rendu par G. Lefebvre, AHRF, t. 29, 1957, p. $272-274$.

(4) Édition de poche publiée par Vintage Books, New York, 1957, 191 p.

(5) Voir G. Lefebvre, «Un Colloque pour l'étude des structures sociales », AHRF, t. 29, 1957, p. $99-105$

(6) Ernest Labrousse (1895-1988), spécialiste de l'histoire économique et sociale de la Révolution.

(7) Marc Bouloiseau (1915-1999), spécialiste de l'histoire de la Révolution, surtout en Normandie. 
Boulogne-s/S, 4-5-58

Cher ami,

Je vous remercie de m'avoir communiqué la table de votre premier volume $^{1}$. Je ne vois rien à y reprendre. Le résultat dépendra du texte que vous publierez. J'observe seulement que je me demande s'il sera question des facteurs qui peuvent suggérer une explication de cette naissance d'une « révolution démocratique ».

J'ajoute que le terme « démocratique » comporte des différences d'interprétation. En 1789, les adversaires d'un ordre nouveau, en l'accusant d'être « démocratique », voulaient dire qu'il substituait au gouvernement d'un monarque ou d'une aristocratie, institué par la Divinité, un gouvernement issu du «démos », c'est-à-dire élu par le peuple. Mais, bien entendu, le mot "démocratique » devint l'objet de discussions lorsqu'on dut déterminer qui aurait le droit de vote - et bien plus encore lorsqu'on se demanda si le gouvernement « démocratique » n'était qu'une « forme » institutionnelle, ou si on lui assignerait la « mission » d'améliorer la condition des membres déshérités de la société, ce qui a ouvert des perspectives illimitées jusqu'au communisme inclus.

Quant à votre appendice $\mathrm{V}^{2}$, j'incline à me ranger en principe derrière Jaurès. Il me paraît en effet que le système adopté par la Constituante était plus libéral, quoique censitaire, que le régime de notre monarchie de 1814 , et d'après votre tableau, plus libéral aussi que le régime anglais, voire américain, à la fin du $18^{\mathrm{e}}$ siècle.

Toutefois, votre exposé appelle une observation importante. Vous ne dites rien en effet de la modalité électorale qui comportait des degrés dans l'opération. C'est un fait d'expérience que l'élection à deux ou plusieurs degrés procure toujours des républicains plus conservateurs, plus favorables aux notables que l'élection au suffrage direct (que Robespierre réclama en vain après le 10 août). Nous savons ici, par le mouvement du Sénat sous la Troisième république, que le suffrage à plusieurs degrés sert de frein à l'élection directe, même sous le régime du suffrage universel.

Or la Constituante adopta l'élection à deux degrés : c'est un fait capital. D'ailleurs on le maintint après le 10 août et sous le Directoire.

Il me paraît que vous pourriez signaler que les Constituants n'oubliaient pas ce qui c'était passé lors des élections aux États-généraux. D'un côté, et vous auriez pu le noter, ils furent moins libéraux que Louis XVI qui avait accordé l'accès aux assemblées paroissiales à tous les contribuables. Ce n'était pas le suffrage universel, comme on le dit par- 
fois ; le plus grand nombre des chefs de famille étaient bien contribuables et des domestiques même payaient la capitation, mais les garçons qui vivaient avec leurs pères n'étaient pas inscrits au rôle fiscal et ne furent donc pas électeurs. Néanmoins la Constituante manifesta clairement une méfiance à l'égard des pauvres bien que, comme je l'ai dit plus haut, le souci de maintenir une certaine union du Tiers contre l'aristocratie, l'ait déterminé à se montrer moins exclusive que les Anglo-saxons.

Toujours est-il (et quand on parle de « révolution bourgeoise »c'est à ce résultat qu'on réfère) que la Constituante ne compta aucun homme du peuple; pourquoi ? Parce que le roi, en accordant un suffrage très étendu, avait spécifié l'élection à plusieurs degrés. Il est inévitable que ce procédé mène au choix de personnages connus par leur influence et leur rôle antérieur ; j'ajoute que les éléments populaires, eux-mêmes, ne sont pas toujours enclins à porter leurs suffrages sur quelqu'un de leur rang (pourquoi lui ? et pas moi ?). Même la Convention n'a compris, à ma connaissance, qu'un seul compagnon de métier (Armonville) $)^{3}$.

Votre examen comparatif me semble donc fondé et je suis tout disposé à le publier ${ }^{4}$; ne vous tracassez pas quant à votre rédaction en français ; elle exige quelques retouches d'expression; j'y procéderai. Mais, à mon avis, il n'y a pas lieu de contester qu'en définitive, malgré la justesse de vos observations, c'est bien la bourgeoisie qui a saisi le pouvoir.

Toutefois, la discussion peut rebondir si on met en cause la structure sociale. Vous avez raison d'estimer que le cens institué par la Constituante, si on prétend qu'il appelait la bourgeoisie au pouvoir, n'impliquait pas de celle-ci une définition si étroite que les très riches seuls fissent partie ; la majorité de la bourgeoisie était «moyenne» et elle pouvait voter et se faire élire. Il en est allé tout autrement à partir de 1814 comme il en allait autrement en Angleterre.

Vous avez d'ailleurs bien raison de juger légère la manière dont les historiens de la Révolution ont évalué le nombre des citoyens actifs et des citoyens passifs et je trouve fort bon que vous le dénombriez.

J'ai passé l'hiver tant bien que mal et, pour le moment, je me trouve assez bien pour supporter les réunions que le bicentenaire de la naissance de Robespierre oblige à tenir. Le travail toutefois doit demeurer assez réduit.

Nous sommes aussi fort préoccupés par votre « récession » car une crise aux États-Unis retentirait chez nous et dans le monde entier. Quant à notre situation politique et à la question algérienne, vous vous doutez bien que mon sentiment ne peut être optimiste. 
Bon courage et bien cordialement

\section{Lefebvre}

(1) R. R. Palmer, The Age of the Democratic Revolution : A Political History of Europe and America, 1760-1800, t. 1, The Challenge, Princeton, Princeton University Press, 1959, 534 p.

(2) Appendice V : Le Caractère « démocratique » et «bourgeois » de la Constitution française de 1791, p. 522-528.

(3) Sur Armonville (1756-1808), voir Gustave LAURENT, « Un Conventionnel ouvrier : JeanBaptiste Armonville », AHRF, t. 1, 1924, p. 217-249, 315-355.

(4) R. R. Palmer, " Sur la composition sociale de la Gauche à la Constituante », $A H R F$, t. 21, 1959, p. 154-156.

Ministère de l'Éducation

Le $12-7-58$

Nationale

Commission Nationale

Pour l'Histoire Économique

de la Révolution

Cher ami,

Vous avez raison de remarquer que les circonstances matérielles et le peu de culture de nombre de citoyens actifs imposaient le vote à deux ou plusieurs degrés. Dans la plupart des bourgs et petites villes de province, ou bien il n'y avait pas d'imprimeurs, ou bien ils ne possédaient qu'un outillage incapable de suffire à une propagande électorale et même de fournir un nombre convenable de bulletins de vote.

Mais sur le caractère conservateur du suffrage à plusieurs degrés, il me faut maintenir mes réflexions. Voyez les élections paroissiales aux États-généraux ; les paysans n'ont participé au choix des députés qu'au second ou au troisième degré ; aucun d'entre eux n'a figuré à la Constituante ; on y voit bien des « cultivateurs » : ce sont des grands propriétaires ruraux qui font valoir. En général, les paysans ont élu des bourgeois. Après le 10 août, on adopte le suffrage universel mais on conserve l'élection à deux degrés malgré Robespierre. La Convention fut toute bourgeoise ; elle ne compta pas de paysans et on n'y trouva qu'un ouvrier [Armonville], choisi par exception à Reims à la faveur des troubles de la Première Terreur. Au surplus, m'en tenant à la France, notre constitution de 1875 , si elle créa un Sénat, ce fut dans la conviction que nommé au scrutin à deux ou trois degrés, il servirait de frein au suffrage universel et ne comporterait que des notables : c'est ce qui arriva. 
J'ai lu avec intérêt votre plaidoyer en faveur de votre dessein. Il me paraît convaincant.

Mais il me faut ajouter que, dans votre conception de la « démocratie », vous ne faites pas une place suffisante à la liberté de l'économie, à la libre entreprise. Ma compétence est principalement française. Il est remarquable que la Déclaration de 1789 ne mentionne pas cette liberté économique. Non que les Constituants ignorassent les économistes du $\mathrm{XVIII}^{\mathrm{e}}$ siècle. Mais ils savaient parfaitement que la grande majorité des Français tenaient à la réglementation : la Constituante se contenta de décréter la liberté du commerce des grains et elle ne réussit pas à la faire respecter. Artisans et boutiquiers tenaient aux corporations. Les Français exécraient la finance (beaucoup de bourgeois aussi d'ailleurs). Sans souffrir encore dans une large mesure de la concentration capitaliste de l'entreprise, ils la détestaient. Ce fut seulement en 1791 que les Constituants sautèrent le pas : ils abolirent les corporations et libérèrent la production ; la Constitution de 1791 enregistra la liberté de l'économie et celle de 1793 fit de même. Ce fut la première fois que sur le Continent la libre entreprise se vit ouvrir la voie. C'est un trait capital de l'œuvre révolutionnaire.

Dans l'esprit des hommes de 89 les conséquences futures n'apparurent ordinairement pas. Comme [Ernest] Labrousse l'a dit, la libération de l'économie parut même «démocratique », comme un équivalent de l'égalité devant la loi : chacun pourrait désormais ouvrir boutique ou former un atelier, et y produire sans aucune entrave.

Reste pourtant que la « démocratie » ainsi conçue ouvrit le champ libre à l'évolution capitaliste déjà commencée et qu'ainsi un fait essentiel du monde moderne, dit « libre », se trouva posé.

Que dire à cet égard des États-Unis ? Je ne pense pas que la liberté économique ait été inscrite dans vos premiers textes constitutionnels. Vous êtes mieux placé que moi pour en parler avec compétence. Mais il n'y a pas de doute, il me semble, que la « démocratie », en Amérique, ait également compris la liberté d'entreprise.

Pour l'Angleterre, elle avait progressé, je pense, dès la première révolution qui supprima beaucoup de monopoles. La Compagnie des Indes subsista toutefois longtemps. Il me paraît que la grande industrie au XVIII ${ }^{\mathrm{e}}$ siècle se développa en toute liberté. Néanmoins, dans beaucoup de villes, je crois savoir que les corporations eurent la vie longue. La complète liberté de l'entreprise fut-elle jamais l'objet d'une proclamation législative ? J'en doute mais ce serait à vérifier. Toujours est-il que là aussi la « démocratie »s'est développée parallèlement à la liberté économique. 
Sur le Continent, il a fallu bien du temps pour qu'elle l'emporte complètement.

Le capitalisme est aujourd'hui sur la défensive.

Il est donc probable que ses partisans n'aiment pas beaucoup qu'on rappelle que la « libre entreprise » l'emporte en même temps que la libération de la pensée, la souveraineté nationale et la « démocratie » politique. Mais c'est un fait et l'historien doit le constater.

Je ne vous dit rien de ce qui se passe ici. Nous tendons le dos. Qu'arrivera-t-il après que le référendum aura ratifié le complot militaire ? Toutes les craintes sont justifiables.

Bien cordialement,

Lefebvre

Boulogne-sur-Seine, $1^{\text {er }}$ juillet [19]59

Cher ami,

Depuis le début d'avril je suis très souffrant. Excès d'urée ; violente crise de foie ; dans le courant de juin, les douleurs se sont calmées ; mais les forces ont disparu et le travail m'est impossible. Je ne puis vous répondre que partiellement ${ }^{1}$.

Sur mes origines familiales et scolaires, ma carrière, mes opinions, ma philosophie, reportez-vous au résumé que j'ai publié dans les Annales en 1947, p. $188^{2}$.

Quand je préparais l'agrégation en 1896-98, [Charles] PetitDutaillis, professeur d'histoire du Moyen âge ${ }^{3}$, me regardait comme un de ses meilleurs étudiants. Le Moyen âge m'a beaucoup servi en effet à l'agrégation. Ensuite il fut question d'une thèse - par exemple sur une des grandes abbayes de Flandre.

Agrégé, nommé au lycée de Cherbourg, mon orientation a changé et s'est tournée vers la Révolution et l'histoire agraire. Influence de Jaurès (Histoire socialiste) et de [Jean] Loutchitsky ${ }^{4}$ dont la méthode comptable marque fortement ma thèse sur les Paysans du Nord préparée de 1904 à 1914, interrompue de 1914 à 1920, reprise en 1920, terminée en $1924^{5}$.

Parallèlement, Jaurès en 1904 avait fait créer la Commission pour la publication des documents inédits sur l'histoire économique de la 
Révolution. Dans quelques départements se formèrent des comités correspondants. Je fus le seul travailleur de celui du Nord. Je réunis un grand nombre de documents sur l'histoire des subsistances. On jugea la liasse trop considérable pour être publiée et on me réduisit au district de Bergues : 2 volumes parus en 1914 et en $1921^{6}$. L'ouvrage est peu connu. L'introduction très considérable fut jugée par [Albert] Mathiez la meilleure étude sur les subsistances et le maximum des grains ${ }^{7}$.

Ces deux directions ne se confondaient pas mais ne se contrariaient pas non plus. Mais en 1907 on introduisit une troisième toute différente. Petit-Dutaillis qui ne m'oubliait pas me proposa de traduire [William] Stubbs ${ }^{8}$. Il eut été raisonnable de refuser une besogne absolument étrangère aux autres. J'ai pourtant accepté afin de gagner un peu d'argent. J'étais très pauvre : marié aussitôt après l'agrégation et bientôt père de deux filles, obligé d'aider mes parents et de contribuer aux études de mon jeune frère (mon cadet de 15 ans) ${ }^{9}$.

Et en 1927 autre complication. Petit[-Dutaillis] m'informa que le troisième volume comporterait 4 études complémentaires ; qu'il ne pouvait exécuter que 2 ; qu'il me fallait me charger de la Curia regis et des Origines du Parlement. À ce moment, j'étais désigné maître de conférences à la Faculté de Clermont. J'ai pourtant accepté, de nouveau pour raison analogue ; ma thèse avait été imprimée en 1924 mais il me fallait payer le coût qui, pour l'époque, était énorme. Rude besogne : j'ai dû lire les livres parus depuis une quinzaine d'années en Angleterre et aux ÉtatsUnis, certainement ceux de [Thomas Frederick] Tout ${ }^{10}$. Je m'en suis tiré ! Des universités anglaises se sont intéressées à mes études qui sont comprises dans le tome III de Stubbs : ils les ont fait traduire et mon texte anglais a été publié dans un volume distinct par 1'Université de Manchester $^{11}$.

Stubbs ne m'a pas enrichi. Je suis resté fort pauvre au moins jusqu'à la cinquantaine. C'est pourquoi je n'ai quitté la France à partir de 1928 - encore, si je suis allé depuis en Angleterre et sur le Rhin, ce fut pour remplir des missions universitaires.

En somme, vous voyez que jusque vers 1928, je me suis partagé entre la Révolution (de plus en plus envahissante) et le Moyen âge représenté par Stubbs. Depuis 1930 je ne suis plus que le spécialiste de la Révolution.

Quand, après tant d'années, je me reporte au passé, je suis surpris que cette période de travail frénétique n'ait pas altéré ma santé. Il n'en fut rien et je n'ai même jamais conçu d'inquiétude. J'ai poussé mes efforts sans broncher durant près de trente ans. 
Entré en 1924 dans l'enseignement supérieur, j'ai continué à beaucoup travailler mais avec plus de variété. J'ai poursuivi les recherches (Grande peur, 1932 ${ }^{12}$; Questions agraires au temps de la Terreur ${ }^{13}$ ) mais j'ai abordé les œuvres de synthèse. Sur cette époque, il me semble que vous êtes suffisamment renseigné.

J'ajoute seulement que Marc Bloch ne fut pour rien dans l'origine et l'exécution de mes ouvrages agraires. Il était plus jeune : au temps de mon grand élan, de 1904 à 1914, il était encore élève à Louis le Grand et à l'École normale. De plus, parisien, il ne pouvait même pas connaître de nom l'obscur érudit provincial. Nous ne nous sommes vus qu'à partir de 1928, lors de mon entrée à la faculté de Strasbourg : ce fut lui qui me fit appeler en invoquant ma thèse parue en 1924 et dont il m'a dit que personne n'avait encore conçu et écrit un livre « comme ça ».

La réimpression en langue française du texte de ma thèse (sans l'apparat critique sans les tableaux statistiques) est due exclusivement à l'initiative des éditeurs de Bari ${ }^{14}$. Aucun éditeur français ne l'aurait entreprise. Je dois beaucoup aux Italiens comme à vous. À Bari on prépare depuis longtemps une traduction de mon Napoléon. Einaudi, à Turin, a publié en 1958 une traduction de ma Révolution, remarquablement illustrée ${ }^{15}$. Il est vrai qu'en 1954, dans mes Études, on avait annoncé des traductions à Barcelone, à Lisbonne et à Cologne. Il n'en a plus été question. On a dû y renoncer, probablement au profit de l'histoire illustrée de la Civilisation, publiée par les Presses universitaires [de France].

J'ai commencé il y a deux jours cette longue lettre. En ce moment, il faut que je m'arrête. Excusez moi : la fatigue m'accable.

\section{Cordialement}

\section{Lefebvre}

(1) Dans cette lettre, G. Lefebvre répond évidemment à des questions posées par R. R. PALmer qui était en train de rédiger un essai sur sa longue carrière. L'article, « Georges Lefebvre : The Peasants and the French Revolution », fut publié dans le Journal of Modern History, t. 31, 1959, p. 329-342.

(2) «Pro domo », $A H R F$, t. 29, 1947, p. 188-190.

(3) Charles Petit-Dutaillis (1868-1947), archiviste-paléologue, spécialiste de l'histoire médiévale.

(4) Jean Loutchitsky (1845-1918), historien russe, spécialiste de l'histoire agraire française. À l'égard de son influence sur G. Lefebvre, voir G. OLIVA, « Georges Lefebvre et les historiens russes de la Révolution française ", $A H R F$, t. 56, 1979, p. 405-406.

(5) Les Paysans du Nord pendant la Révolution française, 2 tomes en 1 vol., Lille, Marquant, 1924, XXV-1020 p. 
(6) Documents relatifs à l'histoire des subsistances dans le district de Bergues pendant la Révolution (1788-an V), 2 vol., Lille, Camille Robbe, 1914-1921. Collection de documents inédits sur l'histoire économique de la Révolution française, t. 1, CXXIV-670 p. ; t. 2, 704 p.

(7) Compte rendu par A. Mathiez, $A H R F$, t. 1, 1924, p. 470-478.

(8) William StubBs (1825-1901), Histoire constitutionnelle de l'Angleterre : son origine et son développement, édition française avec introduction, notes et études historiques inédites par Ch. Petit-Dutaillis ; traduction du texte anglais d'après la dernière édition par G. Lefebvre, Paris, V. Giard et E. Brière, 1907-1927.

(9) Théodore Lefebvre (1889-1943), géographe et professeur à l'Université de Poitiers, exécuté par les Nazis comme Résistant. Sur sa carrière, voir l'article nécrologique, $A H R F$, t. 19, 1947 , p. $68-70$

(10) Thomas Frederick Tout (1855-1929), historien britannique, spécialiste de l'histoire constitutionnelle médiévale.

(11) Studies and Notes Supplementary to Stubbs" "Constitutional History ", vol. 3, by Ch. Petit-Dutalllis and Georges Lefebvre, Manchester, University Press, 1930, Publications of the University of Manchester, Historical Series, n ${ }^{\circ}$ 53, «Additional Studies », p. 348-505.

(12) La Grande Peur de 1789, Paris, Armand Colin, 1932, 272 p.

(13) Questions agraires au temps de la Terreur, Strasbourg, F. LeNIG, 1932, 256 p.

(14) Les Paysans du Nord, deuxième édition, Collezione storica, Bari, Laterza, 1959, 923 p.

(15) La Rivoluzione francese, Turin, G. EINAUdi, 1958, 831 p. 